\title{
Influencia del Hollín sobre la Resistencia a la Compresión de Ladrillos Cerámicos
}

\section{Soot Influence on the Compressive Strength of Ceramic Bricks}

DOI: http://dx.doi.org/10.17981/ingecuc.12.2.2016.10

Artículo de Investigación Científica - Fecha de Recepción: 20 de octubre de 2016, Fecha de Aceptación: 30 de noviembre de 2016

\author{
Alejandro David Martínez Amariz \\ Universidad de Santander. Bucaramanga (Colombia) \\ alejandrom@udes.edu.co \\ Leda Paz Muñoz \\ Universidad de Santander. Bucaramanga (Colombia) \\ lepamu16@udes.edu.co
}

Cómo citar este artículo:

A. Martínez Amariz y L. Paz Muñoz. "Influencia del hollín sobre la resistencia a la compresión de ladrillos cerámicos", INGE CUC, vol. 12, No. 2, pp. 97-102, 2016. DOI: http://dx.doi.org/10.17981/ingecuc.12.2.2016.10

\begin{abstract}
Resumen- Este artículo presenta los resultados obtenidos en las propiedades mecánicas de la adición del hollín, el cual es un subproducto de la cocción de los ladrillos usados en las ladrilleras de Santander. La arcilla usada inicialmente fue caracterizada por el método granulométrico manual siguiendo la norma NSR 10. Las composiciones químicas y mineralógicas se analizaron por medio de la técnica de Difracción de Rayos - X). El análisis de las propiedades mecánicas de los cerámicos se desarrolló mediante el ensayo de compresión según los lineamientos de la Norma Técnica Colombiana NTC: 4017. Los resultados muestran que la adición menor a $60 \%$ en masa de residuos de hollín cuando se adiciona a la arcilla aumenta la capacidad de resistencia a la compresión en un valor promedio de $265.31 \mathrm{kgf} / \mathrm{cm} 2$; este aumento es significativo, ya que el valor mínimo a la compresión según la norma es de $140 \mathrm{kgf} / \mathrm{cm} 2$
\end{abstract}

Palabras clave- Cerámicos, resistencia a la compresión, DRX, hollín sostenibilidad.

\begin{abstract}
This article presents the results obtained in the mechanical properties of the addition of soot, which is a byproduct of cooking the bricks used in Santander brickworks. The clay used was initially characterized using the manual granulometric method, following the NSR 10 standard. Chemical and mineralogical compositions were analyzed using a X-ray Diffraction technique. The analysis of the mechanical properties of ceramic was developed through compression tests, according to the guidelines of the Colombian NTC: 4017 Technical Standard. The results show that adding less than $60 \%$ in mass of soot residues, when clay is added, increases the compressive strength to $265.31 \mathrm{~kg} / \mathrm{cm} 2$ in average. This increase is significant taking into account that the minimum compression value according to the standard is $140 \mathrm{kgf} / \mathrm{cm} 2$.
\end{abstract}

Keywords- Ceramics, compressive strength, XRD, sustainability, soot. 


\section{INTRODUCCIÓN}

La construcción es uno de los sectores más importantes en las economías mundiales; actualmente se busca que esta sea sostenible. Los materiales que son utilizados en las edificaciones son el primer factor que se debe considerar en la búsqueda de construcciones sostenibles. Investigaciones recientes muestran que es posible producir materiales para esta industria con desechos industriales, lo cual busca reducir el impacto ambiental [1].

Uno de los materiales más usados a nivel mundial son los ladrillos cerámicos por sus excelentes propiedades mecánicas y estéticas; no obstante, para su proceso de producción se hacen uso de grandes cantidades de arcilla para su producción, lo cual repercute en un deterioro progresivo del medio ambiente, que se ve reflejado en el aumento de la huella de carbono y que viene acompañado de las emisiones de $\mathrm{CO}_{2}$ por el proceso de cocción del mismo [2].

La explotación de las minas de arcilla se hace a cielo abierto mediante corte de taludes, banqueo y terraceo, lo cual crea cambios en la geomorfología del suelo, inestabilidad, erosión por arrastre de aguas superficiales que forma surcos y cárcavas, sedimentación y cambios del paisaje. La erosión favorece condiciones para movimientos en masa, fenómenos de reptación del suelo y deslizamientos que crean riesgo de desastre para la comunidad junto con el desplazamiento y extinción de especies de fauna y flora de la zona [3].

En la actualidad muchos países investigan y proponen nuevos aditivos para ser usados en el ladrillo cerámico para convertirlo en un producto sostenible a largo plazo, con lo cual se reduciría el consumo de arcilla y con esto cumplir con la tendencia mundial de lograr construcciones sostenibles. Por ejemplo, en Brasil se han estudiado ladrillos a base de arcilla y residuos textiles, los cuales han presentado propiedades mecánicas satisfactorias y de acuerdo con las especificaciones técnicas de las normas de ese país [4].

Diferentes tipos de residuos han sido incorporados con éxito en ladrillos de arcilla cocida. Los residuos y materiales de desecho varían desde los más comúnmente utilizados, como los diversos tipos de cenizas volantes y lodos, hasta aserrín, residuos de pulpa, papel, poliestireno, residuos de té procesados, tabaco, hierba, granos, parabrisas de vidrio, láminas de PVC, papeles de etiquetas, fosfogypsume (residuos usados por plantas de ácido fosfórico), concentrador de boro y colillas de cigarrillos [5].

Colombia no es ajena a esta situación y se han realizado esfuerzos notorios en esta temática. Una iniciativa es la integración de la Arcilla y el Holín para obtener un producto más amigable con el medio ambiente. Idea que nació gracias a la identificación de grandes cantidades de hollín apiladas y abandonadas dentro de las empresas ladrilleras; estos residuos no son almacenados debidamente ni transportados a ningún centro de acopio. En otros países como España existen centros de acopio especializados en la recolección y procesamiento de los residuos de la construcción y demolición [6].

Lo anterior ha motivado a investigar acerca del aprovechamiento de estos residuos controlando su producción, lo cual puede servir de materia prima para la fabricación de nuevos cerámicos, lo cual contribuiría a la creación de sistemas productivos más eficientes y amigables con el medio ambiente.

El objetivo de este trabajo está dirigido a evaluar la propiedad mecánica de compresión de ladrillos cerámicos cuando son fabricados por medio de adiciones de diferentes porcentajes en masa de hollín a la mezcla final. Las probetas usadas fueron desarrolladas mediante diferentes mezclas de arcilla y de hollín, las cuales se usaron con tasas de sustitución de 20, 30, 40, 50 y $60 \%$ de este último. Se realiza una caracterización química y mineralógica de arcilla. Se discuten las cualidades de la arcilla y sus fases mineralógicas.

\section{Metodología}

Las arcillas caracterizadas se obtuvieron en la empresa Cerámica y Ladrillera Ecológica el Estoraque, ubicada en la vereda Lagunetas, sector de La Campiña, jurisdicción del municipio de Girón (Santander); el residuo industrial, Hollín adicionado, provino de la Ladrillera Ergos, ubicada en la vereda Llanadas, jurisdicción del municipio de Girón. La arcilla estaba compacta y en tamaño promedio superior a $16 \mathrm{~mm}$, para lo cual debió ser triturada en la máquina de los ángeles, que consiste en un tambor cilíndrico que gira en posición horizontal y en donde se coloca el material para ser fraccionada por medio de esferas de acero; con esto logra un tamaño máximo promedio de $6 \mathrm{~mm}$ para ser sometida al ensayo de granulometría siguiendo la norma NSC 10 . El hollín presentaba un tamaño nominal de $6 \mathrm{~mm}$, por lo tanto no se requirió triturado. El material de residuo se usó tal y como proviene de los residuos del carbón usado en el proceso de la combustión. Cabe resaltar que el subproducto hollín es una mezcla de carbón y en poco porcentaje arcilla triturada que se desprenden en el propio proceso de cocción.

Para la caracterización mineralógica de las arcillas y el hollín se usó la técnica de DRX de muestras en polvo; las muestras seleccionadas fueron molturadas y homogenizadas en un mortero de ágata y posteriormente montadas en un portamuestra de polimetilmetacrilato (PMMA) mediante la técnica de llenado frontal. El dispositivo usado fue de la marca BRUKER modelo D8 ADVANCE con Geometría Davinci bajo las siguientes condiciones: Voltaje: 40 (Kv); Corriente: 30 (mA); Rendija de Divergencia: $0,6 \mathrm{~mm}$; Rendija Soller Primario: $2.5^{\circ}$; Muestreo: $0.015^{\circ}$ 2theta; Rango de Medición: 3,5 $-70,0^{\circ}$ 
2theta; Radiación: Cuka; Filtro: Níquel; Uso de Antidispersor de Aire: No; Detector: Lineal LynxEye; Tipo de barrido: A pasos; Tiempo de muestreo: 0,4 segundos.

Para el análisis de compresión se prepararon cinco series de mezclas para producir dos probetas por cada una con dimensiones de $30 \times 15 \times 8 \mathrm{~cm}$; estas medidas son las usadas por los ladrillos convencionales usado en la construcción. Para cada ladrillo se tomó una masa base de $5.6 \mathrm{~kg}$, de acuerdo con el normal convencional. La dosificación se realizó en una proporción 1:1 y las composiciones son mostradas en la tabla 1 .

Tabla 1. Composición en Porcentaje (\%) y Masa (GR) de las Diferentes Probetas en el Estudio

\begin{tabular}{|c|c|c|}
\hline PROBETA & ARCILLA (\% - gr) & HOLLIN (\% - gr) \\
\hline $\mathrm{A}$ & $80 \%-4480 \mathrm{gr}$ & $20 \%-1120 \mathrm{gr}$ \\
\hline $\mathrm{B}$ & $70 \%-3920 \mathrm{gr}$ & $30 \%-1680 \mathrm{gr}$ \\
\hline $\mathrm{C}$ & $60 \%-3360 \mathrm{gr}$ & $40 \%-2240 \mathrm{gr}$ \\
\hline $\mathrm{D}$ & $50 \%-2800 \mathrm{gr}$ & $50 \%-2800 \mathrm{gr}$ \\
\hline $\mathrm{E}$ & $40 \%-2240 \mathrm{gr}$ & $60 \%-2240 \mathrm{gr}$ \\
\hline
\end{tabular}

La arcilla se colocó en el recipiente mezclador junto con el hollín y se mezcló para lograr homogeneizar los agregados. Después se añadió agua al proceso hasta obtener una pasta homogénea, y finalmente se llevó la mezcla a la gavera, dispositivo en forma de paralelepípedo que da la forma requerida y en donde se moldeó la probeta en forma de ladrillo macizo; las probetas fueron retiradas y dejadas secar al sol durante 12 horas para eliminar el exceso de humedad; este tipo de preparación es el que se usa de forma artesanal la mayoría de empresas en la región [7]. Este procedimiento fue seguido para las otras mezclas. Las probetas fabricadas fueron sometidos al proceso de cocción a temperatura de 1000 ${ }^{\circ} \mathrm{C}$ aproximadamente en un horno artesanal tipo colmena que opera de forma intermitente y en donde la temperatura y la velocidad de calentamiento son controladas con la alimentación de combustible, que en este caso es carbón mineral; este tipo de ensayo fue realizado en la empresa donde se realizó la investigación. Este tipo de procedimiento también fue realizado por [8]. Se evaluaron las condiciones de resistencia en el laboratorio usando una prensa hidráulica Multitech System MTS 50, la cual produce una carga máxima de hasta 50 toneladas.

\section{III.AnÁlisis y Discusión DE Resultados}

El ensayo de granulometría permitió identificar si los materiales tenían exceso de fracciones gruesas o finas o la presencia de discontinuidades en la distribución por tamaños. En las figuras 1 y 2 se evidencia su distribución granulométrica para el hollín y arcilla, respectivamente.

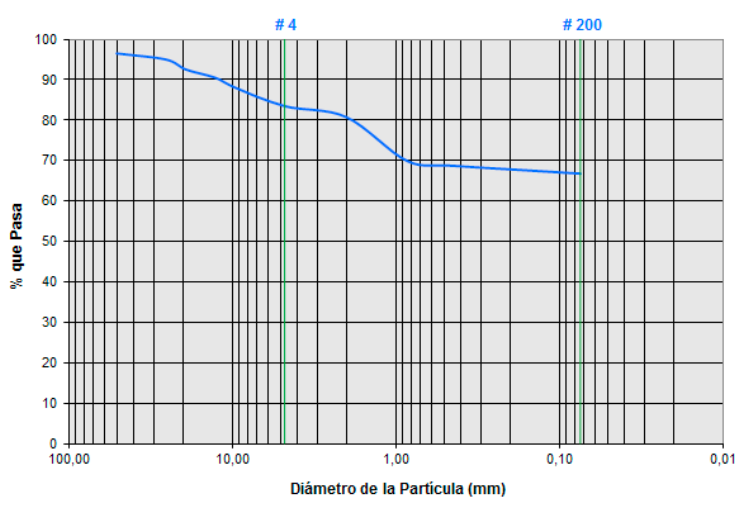

Fig. 1. Distribución granulométrica del hollín Fuente: Autor.

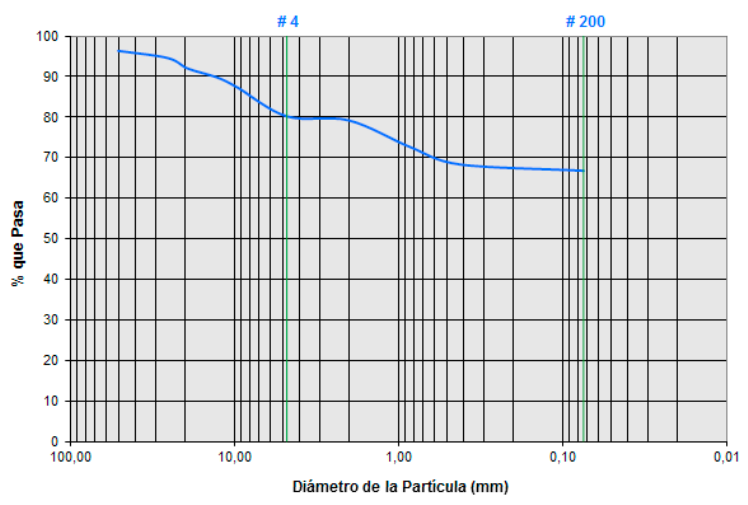

Fig. 2. Distribución granulométrica de la arcilla Fuente: Autor.

Como se ha mencionó, el análisis granulométrico permite definir las fracciones presentes en cuanto a arena fina o gruesa dentro de un árido. Los materiales áridos estudiados en este trabajo presentaron un contenido máximo de arena fina en un $67 \%$ para la arcilla y $67 \%$ para el hollín; este promedio corresponde al porcentaje que pasa por los tamices que corresponden a arena fina según la tabla de numeración y aberturas de tamices propuesta por Espinace [9].

El análisis por DRX para la arcilla y el hollín son presentados en las figuras 3 y 4 . Con base en estos se pueden identificar las fases mayoritarias y minoritarias que componen cada una de las arcillas.

El análisis de los difractógramas se realizó utilizando como patrón de referencia la base de datos del software PCPDFWIN Versión 2.1 JCPDS-ICDD (International Center for Diffraction Data). Considerando la ubicación de los picos, más que su intensidad por la posibilidad de haber favorecido durante la preparación de la muestra ciertos planos cristalinos, se pudo determinar la presencia, principalmente, de las siguientes fases cristalinas, presentadas en la tabla 2 . 


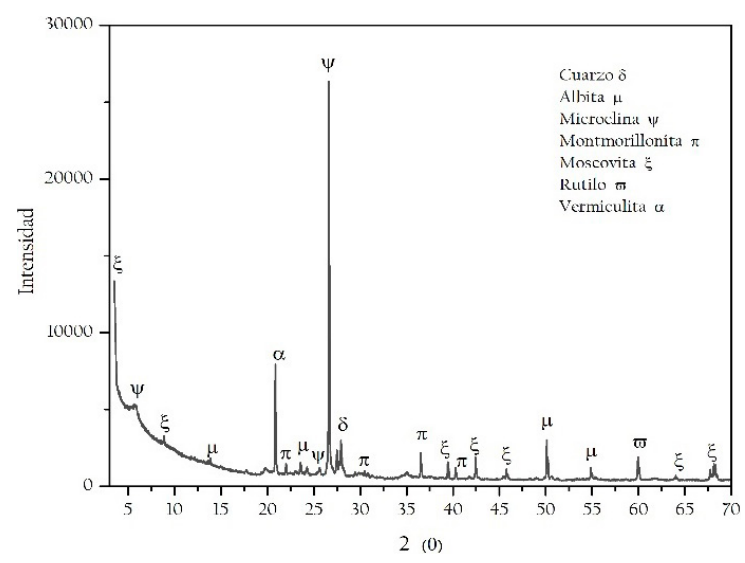

Fig. 3. Difractograma de la arcilla Fuente: Autor.

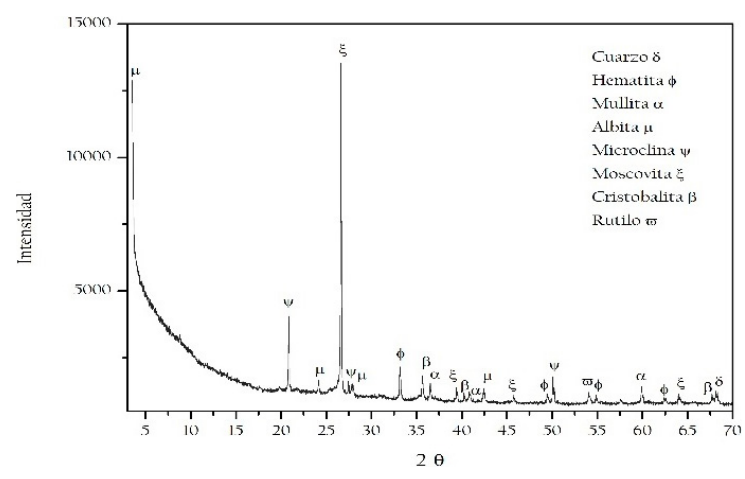

Fig. 4. Difractograma del hollín Fuente: Autor.

Los resultados indican que los minerales arcillosos están constituidos principalmente por aluminosilicatos hidratados con presencia de algunas impurezas, tales como $\mathrm{Na}, \mathrm{Fe}, \mathrm{K}, \mathrm{Ca}$, entre otros, que reiteran los resultados del análisis mineralógico. La tabla II refleja las fases presentes en ambos materiales y destaca el alto contenido de microclina y moscovita presentes en las muestras, lo cual justifica su color amarillo. Asimismo, la variación de los porcentajes que se evidencian en la muestra, pone de manifiesto su tonalidad cremosa. Este tipo de análisis es fundamental para determinar la calidad de las mismas en función del contenido de $\mathrm{SiO}_{2} \mathrm{y} \mathrm{Al}_{2} \mathrm{O}_{3}$ [10]. En el caso del hollín, se evidenció un alto contenido de moscovita y albita, que dan la apariencia de un color café rojizo.

En el ensayo de resistencia a la compresión, las probetas alcanzaron un alto grado de resistencia comparado con la norma NTC 4205, que exige para ladrillos macizos una resistencia a la compresión mínima de $140 \mathrm{Kgf} / \mathrm{cm}^{2}$ para mampostería no estructural tal como se observa en la tabla 3, mientras que la probeta a base de arcilla y hollín presentó en promedio una resistencia a la compresión horizontalmente de $265,31 \mathrm{Kgf} / \mathrm{cm}^{2}$, como se muestra en la tabla 4. La carga fue desplazada y controlada con una tasa de $0,10 \mathrm{~mm} / \mathrm{seg}$, humedad del $63 \%$ y temperatura de $24^{\circ} \mathrm{C}$.

Este estudio guarda relación con un estudio realizado en la República Checa por V. Nežerka et al. [11]; de otra parte, estudios realizados en Ocaña con diferentes tipos de arcillas para la fabricación de ladrillos cerámicos muestran valores menores que el presentado en el nuestro [12].

Esta alta elevada propiedad a la compresión se puede explicar debido a que el hollín puede estar ubicado en los poros o intersticios de la arcilla, lo cual permite que aumente la dureza del material; esto coincide con otros estudios realizados por Silva et al. en los que se incorporó agregados finos de productos descartables de arcillas a morteros de cemento [13] no obstante estudios más a fondo son necesarios.

Tabla 2. Fases Cristalinas de los Materiales

\begin{tabular}{|c|c|c|c|c|}
\hline \multicolumn{5}{|l|}{ Fases cristalinas } \\
\hline Nombre & Nomenclatura & PDF & $\begin{array}{c}\text { Arcilla } \\
\text { (D5YU) }\end{array}$ & Hollín (D5YV) \\
\hline Cuarzo & $\mathrm{Si} \mathrm{O} 2$ & 000-87-2096 & $\mathrm{X}$ & $\mathrm{X}$ \\
\hline Albita & $\mathrm{Na}(\mathrm{Al} \mathrm{Si} 3 \mathrm{O} 8)$ & 000-76-1819 & $\mathrm{x}$ & $\mathrm{x}$ \\
\hline Microclina & K ( i0.75 Al0.25 4 O8) & 000-77-0135 & $\mathrm{X}$ & $\mathrm{X}$ \\
\hline Montmorillonita & $\mathrm{Ca} 0.2(\mathrm{Al}, \mathrm{Mg} 2 \mathrm{Si} 4 \mathrm{O} 10(\mathrm{OH}) 2 ! 4 \mathrm{H} 2 \mathrm{O}$ & 000-13-0135 & $\mathrm{X}$ & \\
\hline Moscovita & K Al3 Si3 O10 (OH)2 & 000-84-1302 & $\mathrm{x}$ & $\mathrm{x}$ \\
\hline Rutilo & Ti O2 & 000-78-1508 & $\mathrm{X}$ & $\mathrm{x}$ \\
\hline Vermiculita & Mg3 Si4 O10 (OH)2 & 000-74-1732 & $\mathrm{X}$ & \\
\hline Hematita & $\mathrm{Fe} 2 \mathrm{O} 3$ & 000-79-1741 & & $\mathrm{x}$ \\
\hline Mullita & Al4.56 Si1.44 O9.72 & 000-79-1458 & & $\mathrm{X}$ \\
\hline Cristobalita & $\mathrm{Si} \mathrm{O} 2$ & 000-82-0512 & & $\mathrm{X}$ \\
\hline
\end{tabular}


Tabla III. Parámetros de Resistencia a la COMPREnsión de LAS UNIDADES DE MAMPOSTERÍA ESTRUCTURAL Y NO ESTRUCTURAL

\begin{tabular}{|l|c|c|c|c|}
\hline Tipo & \multicolumn{2}{|c|}{$\begin{array}{c}\text { Estructural } \\
\text { resistencia mínima } \\
\text { a la compresión } \\
\text { mpa(kgf/cm }\end{array}$} & \multicolumn{2}{|c|}{$\begin{array}{c}\text { No estructural } \\
\text { resistencia mínima } \\
\text { a la compresión } \\
\left.\text { mpa(kgf/cm }{ }^{2}\right)\end{array}$} \\
\hline $\begin{array}{l}\text { Unidad de } \\
\text { mampostería } \\
\text { maciza } \\
\text { (Ladrillo) }\end{array}$ & $\begin{array}{c}20,0 \\
(200)\end{array}$ & $\begin{array}{c}15,0 \\
(150)\end{array}$ & $\begin{array}{c}14,0 \\
(140)\end{array}$ & $\begin{array}{c}10,0 \\
(100)\end{array}$ \\
\hline
\end{tabular}

Esta tabla muestra las conversiones de unidades de resistencia exigidas por la norma, en donde se tiene que $1 \mathrm{kgf} / \mathrm{cm} 2=98067 \mathrm{~Pa}=0.098067 \mathrm{MPa}$.

Tabla 4. Composiciones Ensayadas vs. Resistencia a la Compresión

\begin{tabular}{|c|c|}
\hline $\begin{array}{c}\text { Especímenes } \\
\text { (composiciones) }\end{array}$ & $\begin{array}{c}\text { Resistencia mínima a la } \\
\text { compresión pa }\left(\mathrm{kgf} / \mathrm{cm}^{2}\right)\end{array}$ \\
\hline $\mathrm{A}$ & 272.09 \\
\hline $\mathrm{B}$ & 271.01 \\
\hline $\mathrm{C}$ & 268.67 \\
\hline $\mathrm{D}$ & 272.14 \\
\hline $\mathrm{E}$ & 242.66 \\
\hline Promedio & 265.31 \\
\hline
\end{tabular}

En este estudio, la composición que contenía 50 \% de hollín, la probeta $\mathrm{D}$ fuera mucho más fuerte en comparación con las demás composiciones. Es posible que esta sea la causa de que el agrietamiento se haya producido en las partes superior o inferior de los cerámicos, tal como se muestra en la fig. 5 y que a su vez el porcentaje contenido de arcilla en las composiciones sea el responsable de una mayor permeabilidad y mejor resistencia mecánica.

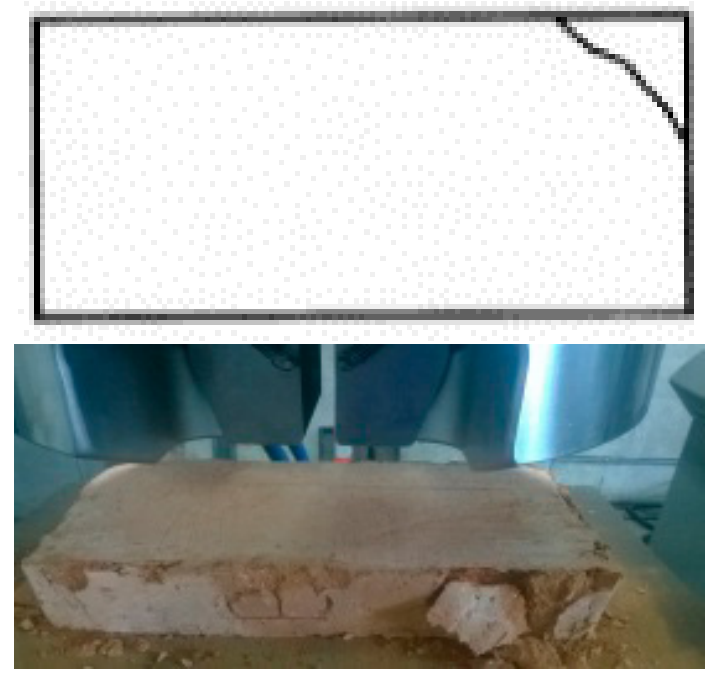

Fig. 5. Falla tipo 5. Fracturas en los lados en las partes superior o inferior (ocurre comúnmente con cabezales no adheridos) Fuente: NTC 673.
De otra parte, queda en evidencia que las adiciones de hollín hasta $60 \%$ de la masa total del ladrillo generan un efecto negativo en la compresión, debido a la introducción de inhomogeneidades en el material, como es observado en el valor del espécimen $\mathrm{E}$.

La fractura que predominó en los diferentes especímenes fue la tipo 5: fracturas en los lados en las partes superior o inferior (ocurre comúnmente con cabezales no adheridos); esta afirmación está basada en el esquema de los modelos de fractura típicos de la NTC 673 [14].

\section{Conclusiones}

Los resultados de los ensayos de granulometría indican que la muestra de $200 \mathrm{~g}$ tiene un índice más alto de arena fina (95\%); resultado de gran importancia para la fabricación de las pastas cerámicas. Este resultado permite clasificar esta muestra como arcilla de alta plasticidad.

Los resultados del análisis mineralógico indican que la arcilla está constituida principalmente por aluminosilicatos hidratados con presencia de algunas impurezas, tales como $\mathrm{Na}$, $\mathrm{Fe}, \mathrm{K}, \mathrm{Ca}$, entre otros, y el hollín, por ser un residuo industrial del proceso de cocción de los ladrillos, demostró contener fases similares a la de la arcilla, diferenciándose por la presencia de compuestos propios del carbón mineral, como lo es la mullita.

Los resultados del análisis de compresión permiten evidenciar que la arcilla es factor importante en la homogeneización de la mezcla, pues los especímenes que alcanzaron una mayor resistencia fueron los que contenían mayor porcentaje de arcilla, es decir, este material le brinda mayor permeabilidad a la mezcla, alcanzando así una resistencia promedio de $265,31 \mathrm{Kgf} / \mathrm{cm}^{2}$ en un $52,77 \%$ por encima del adobe comprimido macizo convencional.

En síntesis, estos resultados abren un amplio campo a futuro para el estudio de nuevas adiciones para la creación y fabricación de ladrillos cerámicos reutilizando materiales descartables del mismo proceso de sinterización; además ayuda a mitigar en gran medida la cantidad de este desecho industrial que genera un alto impacto en el medio ambiente.

\section{Agradecimientos}

Los autores agradecen al Departamento de Ciencia y Tecnología, Colciencias, por el apoyo económico en el marco de la convocatoria de Jóvenes Investigadores 2015. 


\section{Financiación}

Artículo de investigación derivado del proyecto "Caracterización estructural de arcillas usadas en la industria de la construcción para evaluación de sus propiedades físico-mecánicas", financiado por el Departamento de Ciencia y Tecnología, Colciencias, y la Universidad de Santander. Fecha de inicio: 11 de febrero de 2015. Fecha de finalización: 11 de noviembre de 2015 .

\section{REFERENCIAS}

[1] P. Muñoz Velasco, M. P. Morales Ortíz, M. A. Mendívil Giró, and L. Muñoz Velasco, "Fired clay bricks manufactured by adding wastes as sustainable construction material - A review," Constr. Build. Mater., vol. 63 pp. 97-107, 2014. https://doi.org/10.1016/j.conbuildmat.2014.03.045

[2] X. Yan and Y. Fang, "CO2 emissions and mitigation potential of the Chinese manufacturing industry," $J$. Clean. Prod., vol. 103, pp. 759-773, 2015. http://dx.doi. org/10.1016/j.jclepro.2015.01.051

[3] M. S. Silva and L. A. Z. Valencia, "Impacto Ambiental y Gestión del Riesgo de Ladrilleras en la vereda Los Gómez de Itagüí,” Cuad. Act., vol. 0, no. 5, pp. 109-123, 2013.

[4] L. C. S. Herek, C. E. Hori, M. H. Miranda Reis, N Diaz Mora, C. R. Granhem Tavares, and R. Bergamas co, "Characterization of ceramic bricks incorporated with textile laundry sludge," Ceram. Int., vol. 38, no. 2, pp. 951-959, 2012. http://dx.doi.org/10.1016/j.ceramint.2011.08.015

[5] A. A. Kadir and A. Mohajerani, "Bricks: an excellent building material for recycling wastes - a review. Proceedings of the IASTED International Conference", Environmental Management and Engineering. EME 2011.
[6] Catálogo Europeo de Residuos CER, aprobado por la Decisión 2000/532/CE de la Comisión, de 3 de mayo, modificada por las Decisiones de la Comisión, Decisión 2001-118, de 16 de enero, Decisión 2001-119, de 22 de enero, y por la Decisión del Consejo Decisión 573-2001, de 23 de julio [Online]. Disponible en: http://www.ingurumena.ejgv. euskadi.eus/contenidos/estadistica/residuos_rnp/es_res_ rnp/adjuntos/CER_2002.pdf

[7] J. Santos, P. Malagón, "Estudio del mejoramiento del proceso de fabricación de tejas y ladrillos con las arcillas de la vereda Guayabal del municipio de Barichara (Santander)". [Trabajo de Grado], Universidad Industrial de Santander. 2009

[8] J. Camacho and E. Camargo, "Estudios de mezclas de arcillas del norte de Santander para fabricar materiales de construcción". [Trabajo de Grado], Universidad Industrial de Santander, 1986.

[9] R. Espinace, "Tabla de numeración y abertura de tamices", Escuela de Ingeniería y Construcción - Ensayos de Laboratorio. Pontificia Universidad Católica de Valparaíso. [Online], Disponible en: http://icc.ucv.cl/geotecnia/03_ docencia/02_laboratorio/manual_laboratorio/granulometria.pdf

[10] G. Pérez and Y. Vargas, "Desarrollo, de cerámicos refractarios silico-aluminosos a partir de arcillas caoliníticas de Barichara (Santander)". [Trabajo de Grado], Universidad Industrial de Santander, 2009.

[11] V. Nežerka, Z. Slížková, P. Tesárek, T. Plachý, D. Frankeová, and V. Petráňová, "Comprehensive study on mechanical properties of lime-based pastes with additions of metakaolin and brick dust," Cem. Concr. Res., vol. 64, pp. 17-29, 2014. https://doi.org/10.1016/j.cemconres.2014.06.006

[12] C. Piscal Arevalo, N. Afanador García y S. Medina "Resistencia a la compresión en el municipio de Ocaña", Ingenio, vol.4,no. 2, pp. 12-17, 2012.

[13] J. Silva, J. de Brito, and R. Veiga, "Incorporation of fine ceramics in mortars," Constr. Build. Mater., vol. 23, no. 1, pp. 556-564, 2009. https://doi.org/10.1016/j.conbuildmat.2007.10.014

[14] NORMA NTC 673, Ensayo de resistencia a la compresión de especímenes cerámicos. Bogotá, D.C.: Normas Técnicas Colombianas (ICONTEC), 2010. 